\title{
A Phosphole Oxide-Containing Organogold(III) \\ Complex for Solution-Processable Resistive Memory Devices with Ternary Memory Performances
}

Eugene Yau-Hin Hong, Chun-Ting Poon and Vivian Wing-Wah Yam*

Institute of Molecular Functional Materials [Areas of Excellence Scheme,

University Grants Committee (Hong Kong)] and Department of Chemistry,

The University of Hong Kong, Pokfulam Road, Hong Kong, P. R. China

E-mail: wwyam@hku.hk

Supporting Information 


\section{Experimental Section}

Materials and Reagents. All commercially available reagents were of analytical grade and were used as received. All solvents were purified and distilled using standard procedures before use. $\quad\left[\mathrm{Au}\left({ }^{t} \mathrm{BuC}{ }^{\wedge} \mathrm{N}^{\wedge} \mathrm{C}^{t} \mathrm{Bu}\right) \mathrm{Cl}\right]^{1}$ and 2-(triisopropylsilylethynyl)benzo[b]phosphole $P$-oxide ${ }^{2}$ were synthesized according to literature procedures. Tetra- $n$-butylammonium hexafluorophosphate was recrystallized at least three times from absolute ethanol before use.

Physical Measurements and Instrumentation. ${ }^{1} \mathrm{H}$ and ${ }^{31} \mathrm{P}\left\{{ }^{1} \mathrm{H}\right\}$ NMR spectra were recorded on a Bruker AV 400 NMR spectrometer with chemical shifts reported relative to tetramethylsilane and $85 \%$ phosphoric acid respectively. Fast atom bombardment (FAB) mass spectrum was recorded using a Thermo Scientific DFS High Resolution Magnetic Sector Mass Spectrometer. Elemental analyses for the metal complex were performed on the Carlo Erba 1106 elemental analyzer at the Institute of Chemistry, Chinese Academy of Sciences in Beijing. Thermogravimetric analysis (TGA) was performed on a TA Instruments TGA Q50 thermogravimetric analyzer with a heating rate of $10{ }^{\circ} \mathrm{C} \min ^{-1}$ under a nitrogen atmosphere. UV-Vis spectra were obtained on a Varian Cary $50 \mathrm{UV}$-vis spectrophotometer. Steady-state excitation and emission spectra at room temperature and at $77 \mathrm{~K}$ were recorded on a Spex Fluorolog-3 model FL3-211 fluorescence spectrofluorometer equipped with an R2658P PMT detector. Photophysical measurements in low temperature glass were carried out with the sample solution loaded in a quartz tube inside a quartz-walled Dewar flask. Liquid nitrogen was placed into the Dewar flask for low temperature (77 K) photophysical measurements. Excited-state lifetime measurements were performed 
using a conventional laser system. The excitation source used was the $355-\mathrm{nm}$ output (third harmonic, $8 \mathrm{~ns}$ ) of a Spectra-Physics Quanta-Ray Q-switched GCR-150 pulsed Nd:YAG laser $(10 \mathrm{~Hz})$. Luminescence quantum yields were measured by the optical dilute method reported by Demas and Crosby. ${ }^{3}$ A degassed aqueous solution of quinine sulfate in $1.0 \mathrm{~N}$ sulfuric acid (excitation wavelength $=365 \mathrm{~nm}, \Phi=0.546$ ) was used as the reference and corrected for the refractive index of the solution. ${ }^{4}$ All solutions for emission lifetime and luminescence quantum yield studies were degassed on a high-vacuum line in a two-compartment cell consisting of a 10-ml Pyrex bulb and a 1-cm path length quartz cuvette and sealed from the atmosphere by a Bibby Rotaflo HP6 Teflon stopper. The solutions were rigorously degassed with at least four successive freeze-pump-thaw cycles. Cyclic voltammetric measurements were performed by using a $\mathrm{CH}$ Instruments, Inc. model $\mathrm{CHI} 620 \mathrm{E}$ electrochemical analyzer. The electrolytic cell used was a conventional two-compartment cell. Electrochemical measurements were performed in dichloromethane solutions with 0.1 $\mathrm{M}^{n} \mathrm{Bu}_{4} \mathrm{NPF}_{6}$ as supporting electrolyte at room temperature. The reference electrode was a $\mathrm{Ag} / \mathrm{AgNO}_{3}(0.1 \mathrm{M}$ in acetonitrile $)$ electrode, and the working electrode was a glassy carbon electrode ( $\mathrm{CH}$ Instruments, Inc.) with a platinum wire as the counter electrode in a compartment separated from the working electrode by a sintered-glass frit. The ferrocenium/ferrocene couple $\left(\mathrm{FeCp}_{2}{ }^{+/ 0}\right)$ was used as the internal reference. ${ }^{5}$ All solutions for electrochemical studies were deaerated with pre-purified argon gas before measurement.

Fabrication and Characterization of the Memory Device. The memory devices were fabricated on an indium-tin oxide (ITO)-coated glass substrate which was pre-cleaned by sonicating successively with deionized water, acetone, isopropanol 
and absolute ethanol for 15 minutes each. A chloroform solution of complex 1 (5 mg $\mathrm{ml}^{-1}$ ) was spin-coated onto the ITO glass substrate. The thin film was baked on a hot plate at $70{ }^{\circ} \mathrm{C}$ for $10 \mathrm{~min}$. Aluminum top electrodes were thermally evaporated and deposited onto the films through a shadow mask at a pressure of $c a .5 \times 10^{-6}$ mbar. Devices with area of $c a .0 .25 \mathrm{~mm}^{2}$ were obtained. The devices were characterized under ambient conditions, in a probe station equipped with a Keithley 4200-SCS semiconductor characterization system.

Electron Microscopy. SEM experiments were performed on a Hitachi S-4800 FEG scanning electron microscope at the Electron Microscope Unit of The University of Hong Kong.

Atomic Force Microscopy. AFM images were obtained using a MFP-3D atomic force microscope (Asylum Research) in tapping mode with a scan rate of $1.0 \mu \mathrm{m} \mathrm{s}^{-1}$. The sample was prepared by spin-coating a chloroform solution of complex 1 (5 mg $\mathrm{ml}^{-1}$ ) onto a quartz plate which was then dried in air before measurement.

\section{Synthesis and Characterization}
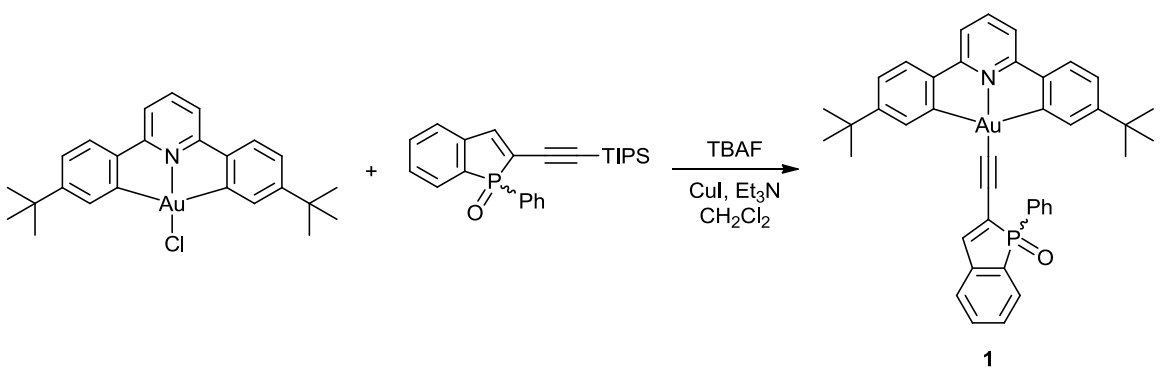

Complex 1. This was synthesized according to modification of a literature procedure for the preparation of $\left[\mathrm{Au}\left(\mathrm{C}^{\wedge} \mathrm{N}^{\wedge} \mathrm{C}\right)(\mathrm{C} \equiv \mathrm{CPh})\right] .{ }^{6}$ To a stirred solution of 
2-(triisopropylsilylethynyl)benzo[b]phosphole $P$-oxide $(122 \mathrm{mg}, 0.30 \mathrm{mmol})$ and $\left[\mathrm{Au}\left({ }^{t} \mathrm{BuC}{ }^{\wedge} \mathrm{N}^{\wedge} \mathrm{C}^{t} \mathrm{Bu}\right) \mathrm{Cl}\right](105 \mathrm{mg}, 0.18 \mathrm{mmol})$ in the presence of a catalytic amount of copper(I) iodide in triethylamine $(0.2 \mathrm{ml})$ and dichloromethane $(30 \mathrm{ml})$ at $0{ }^{\circ} \mathrm{C}$ was added tetra- $n$-butylammonium fluoride solution (1.0 M in THF, $0.3 \mathrm{ml}, 0.30 \mathrm{mmol}$ ). The reaction mixture was stirred at $0{ }^{\circ} \mathrm{C}$ under nitrogen and the progress was monitored by TLC. The mixture was then filtered and evaporated to dryness under reduced pressure. The crude product was purified by flash column chromatography on silica gel using dichloromethane-acetone $(9: 1 \mathrm{v} / \mathrm{v})$ as eluent to give a yellow solid. Recrystallization by layering of hexane onto a concentrated chloroform solution yielded the complex as yellow crystals. Yield: $112 \mathrm{mg}(78 \%) .{ }^{1} \mathrm{H}$ NMR (400 MHz, $\left.\mathrm{CDCl}_{3}, 298 \mathrm{~K}\right): \delta 1.32\left(\mathrm{~s}, 18 \mathrm{H},{ }^{t} \mathrm{Bu}\right), 7.18\left(\mathrm{dd}, J=8.2,2.0 \mathrm{~Hz}, 2 \mathrm{H}\right.$, phenyl of $\left.\mathrm{C}^{\wedge} \mathrm{N}^{\wedge} \mathrm{C}\right)$, 7.28-7.39 (m, 6H, phenyl of $\mathrm{C}^{\wedge} \mathrm{N}^{\wedge} \mathrm{C}$, pyridine of $\mathrm{C}^{\wedge} \mathrm{N}^{\wedge} \mathrm{C}$ and phenyl of benzophosphole oxide), 7.39-7.55 (m, 5H, phenyl of benzophosphole oxide), 7.62 ( $\mathrm{t}$, $J=8.4 \mathrm{~Hz}, 1 \mathrm{H}$, phenyl of benzophosphole oxide), $7.77(\mathrm{t}, J=8.0 \mathrm{~Hz}, 1 \mathrm{H}$, pyridine of $\left.\mathrm{C}^{\wedge} \mathrm{N}^{\wedge} \mathrm{C}\right), 7.86-7.95(\mathrm{~m}, 2 \mathrm{H}$, phenyl of benzophosphole oxide), 7.97 (d, $J=2.0 \mathrm{~Hz}, 2 \mathrm{H}$, phenyl of $\left.\mathrm{C}^{\wedge} \mathrm{N}^{\wedge} \mathrm{C}\right) .{ }^{31} \mathrm{P}\left\{{ }^{1} \mathrm{H}\right\} \mathrm{NMR}\left(162 \mathrm{MHz}, \mathrm{CDCl}_{3}, 298 \mathrm{~K}\right): \delta$ 36.96. Positive FAB-MS: $m / z, 788\left([\mathrm{M}]^{+}\right)$. Elemental analysis calcd $(\%)$ for $\mathrm{C}_{41} \mathrm{H}_{37} \mathrm{AuNOP} \cdot \mathrm{H}_{2} \mathrm{O}: \mathrm{C}$, 61.12; H, 4.87; N, 1.74; found: C, 61.41; H, 4.57; N, 1.78. 


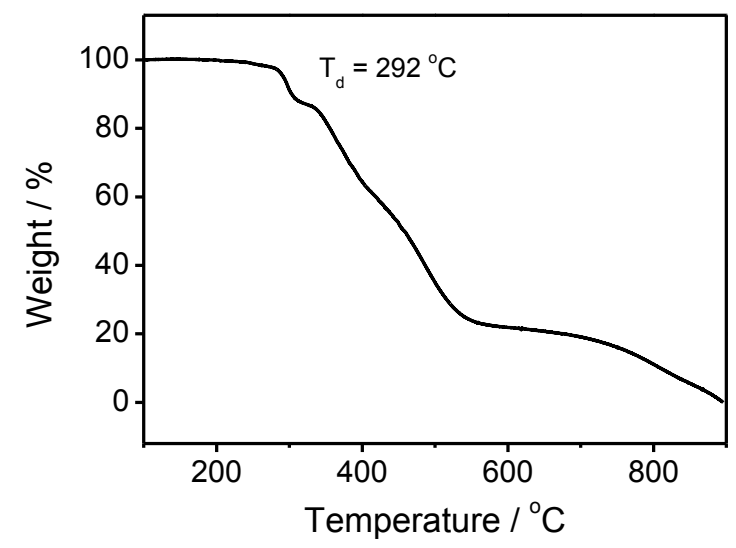

Figure S1. TGA thermogram of complex 1. Heating rate $=10{ }^{\circ} \mathrm{C} \mathrm{min}{ }^{-1}$ under a nitrogen atmosphere. Decomposition temperature $\left(T_{\mathrm{d}}\right)$ is defined as the temperature at which the material shows a $5 \%$ weight loss. 


\section{Crystallographic Data}

Table S1 Crystal and structure determination data of complex 1

\begin{tabular}{ll}
\hline Empirical formula & $\mathrm{C}_{41} \mathrm{H}_{37} \mathrm{AuNOP}$ \\
Formula weight & 787.65 \\
Temperature & $296(2) \mathrm{K}$ \\
Wavelength & $0.71073 \AA$ \\
Crystal system & Monoclinic \\
Space group & $\mathrm{P} 2{ }_{1} / \mathrm{c}$ \\
Unit cell dimensions & $a=15.935(3) \AA$ \\
& $b=13.013(3) \AA$ \\
& $c=17.978(4) \AA$ \\
Volume & $3449.8(13) \AA^{3}$ \\
$Z$ & 4 \\
Density (calculated) & $1.517 \mathrm{mg} \mathrm{m}^{-3}$ \\
Absorption coefficient & $4.343 \mathrm{~mm}^{-1}$ \\
$F_{000}$ & 1568 \\
Crystal size & $0.400 \mathrm{~mm}^{\circ} \times 0.300 \mathrm{~mm} \times 0.200 \mathrm{~mm}$ \\
$\theta$ range for data collection & 1.987 to $25.249^{\circ}$ \\
Index ranges & $-19 \leq h \leq 18,-15 \leq k \leq 15,-21 \leq l \leq 21$ \\
Reflections collected & 27251 \\
Independent reflections & $6195\left[R_{\text {int }}=0.0622\right]$ \\
Completeness to $\theta=25.242^{\circ}$ & $99.0 \%$ \\
Absorption correction & Multi-scan \\
Max. and min. transmission & 0.7457 and 0.5660 \\
Refinement method & Full-matrix least-squares on $F^{2}$ \\
Data / restraints / parameters & $6195 / 42 / 462$ \\
Goodness-of-fit on $F^{2}$ & 1.092 \\
Final $R$ indices $[I>2 \sigma(I)]$ & $R_{1}=0.0342, w R_{2}=0.0840$ \\
$R$ indices (all data) & $R_{1}=0.0541, w R_{2}=0.0892$ \\
Large diff. peak and hole & 0.707 and $-0.795 \mathrm{e} \AA^{-3}$ \\
\hline
\end{tabular}


Table S2 Selected bond lengths $(\AA)$ and angles $\left(^{\circ}\right)$ of complex 1 with estimated standard deviation (esds) in parentheses

\begin{tabular}{|ll|ll|}
\multicolumn{5}{c}{ Bond length $(\AA)$} \\
$\mathrm{Au}(1)-\mathrm{C}(1)$ & $2.087(6)$ & $\mathrm{C}(26)-\mathrm{C}(27)$ & $1.194(7)$ \\
$\mathrm{Au}(1)-\mathrm{C}(26)$ & $1.972(6)$ & $\mathrm{P}(1)-\mathrm{O}(1)$ & $1.467(4)$ \\
$\mathrm{Au}(1)-\mathrm{N}(1)$ & $2.011(4)$ & $\mathrm{P}(1)-\mathrm{C}(28)$ & $1.824(6)$ \\
$\mathrm{N}(1)-\mathrm{C}(7)$ & $1.347(7)$ & $\mathrm{P}(1)-\mathrm{C}(36)$ & $1.809(6)$ \\
\multicolumn{4}{c}{ Bond angle $\left(^{\circ}\right)$} \\
$\mathrm{C}(1)-\mathrm{Au}(1)-\mathrm{C}(17)$ & $162.1(2)$ & $\mathrm{C}(2)-\mathrm{C}(1)-\mathrm{Au}(1)$ & $129.2(4)$ \\
$\mathrm{N}(1)-\mathrm{Au}(1)-\mathrm{C}(26)$ & $177.4(2)$ & $\mathrm{Au}(1)-\mathrm{C}(26)-\mathrm{C}(27)$ & $175.3(5)$ \\
$\mathrm{C}(1)-\mathrm{Au}(1)-\mathrm{N}(1)$ & $80.7(2)$ & $\mathrm{C}(27)-\mathrm{C}(28)-\mathrm{P}(1)$ & $121.8(4)$ \\
$\mathrm{C}(1)-\mathrm{Au}(1)-\mathrm{C}(26)$ & $96.8(2)$ & $\mathrm{O}(1)-\mathrm{P}(1)-\mathrm{C}(36)$ & $111.3(3)$ \\
$\mathrm{C}(7)-\mathrm{N}(1)-\mathrm{Au}(1)$ & $117.4(4)$ & $\mathrm{O}(1)-\mathrm{P}(1)-\mathrm{C}(28)$ & $118.0(3)$ \\
$\mathrm{C}(6)-\mathrm{C}(1)-\mathrm{Au}(1)$ & $110.5(5)$ & $\mathrm{C}(28)-\mathrm{P}(1)-\mathrm{C}(35)$ & $92.2(3)$
\end{tabular}

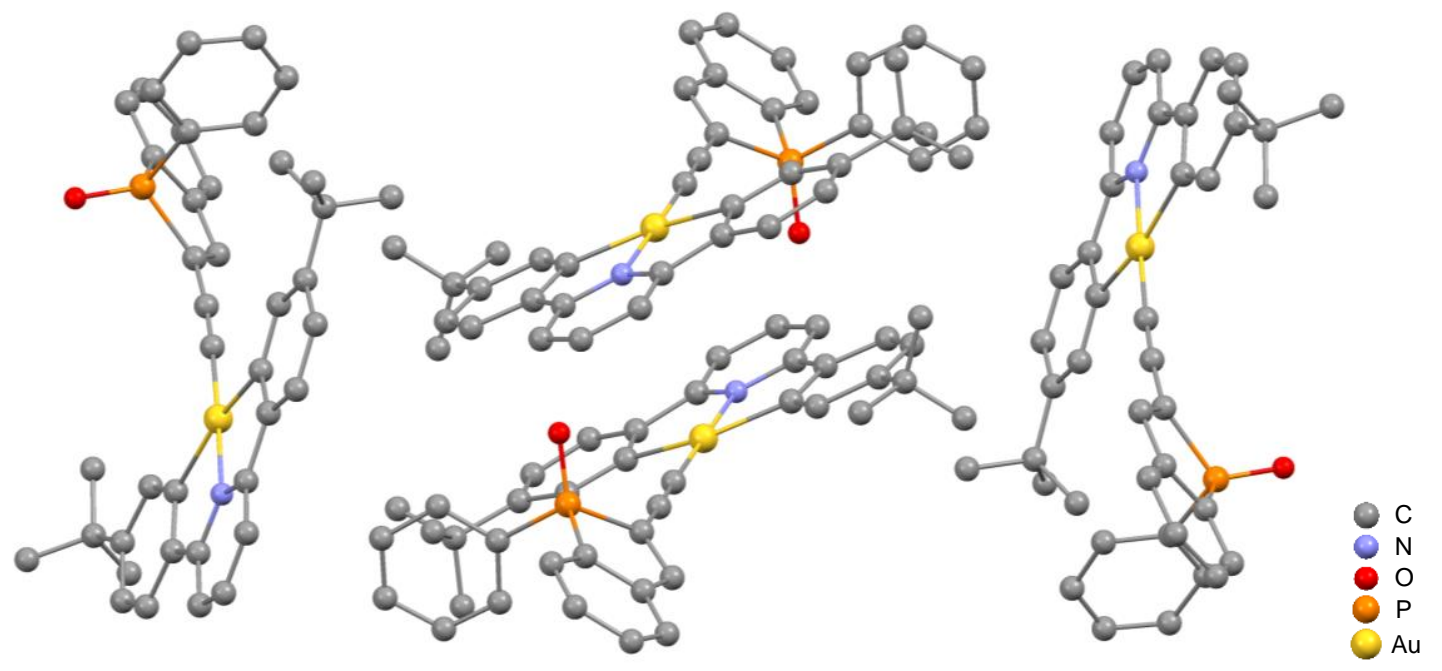

Figure S2. Crystal packing of complex 1. 


\section{Photophysical Data}

Table S3 Photophysical data of complex 1

\begin{tabular}{|c|c|c|c|}
\hline Absorption $^{a}$ & \multicolumn{3}{|c|}{ Emission } \\
\hline$\lambda_{\max } / \mathrm{nm}\left(\varepsilon_{\max } / \mathrm{dm}^{3} \mathrm{~mol}^{-1} \mathrm{~cm}^{-1}\right)$ & $\operatorname{Medium}(T / \mathrm{K})$ & $\lambda_{\max } / \mathrm{nm}\left(\tau_{0} / \mu \mathrm{s}\right)$ & $\Phi_{\text {lum }}^{b}$ \\
\hline \multirow{4}{*}{$\begin{array}{l}304 \text { (20965), } 315 \text { (20785), } 361 \text { (18260), } \\
384 \text { (14775), } 413 \text { (5560) }\end{array}$} & $\mathrm{CH}_{2} \mathrm{Cl}_{2}$ (298) & $454(<0.1)$ & $2.9 \times 10^{-4}$ \\
\hline & & $651,708 \mathrm{sh}(4.69)$ & \\
\hline & glass $(77)^{c}$ & $437(<0.1)$ & \\
\hline & & $482,513,549 \operatorname{sh}(256)$ & \\
\hline
\end{tabular}

${ }^{a}$ Measured in dichloromethane solution at $298 \mathrm{~K} .{ }^{b}$ Luminescence quantum yield, measured at room temperature using quinine sulfate in $1.0 \quad \mathrm{~N} \quad \mathrm{H}_{2} \mathrm{SO}_{4}$ as a standard. ${ }^{c}$ Measured in ethanol-methanol-dichloromethane (40:10:1 v/v/v).

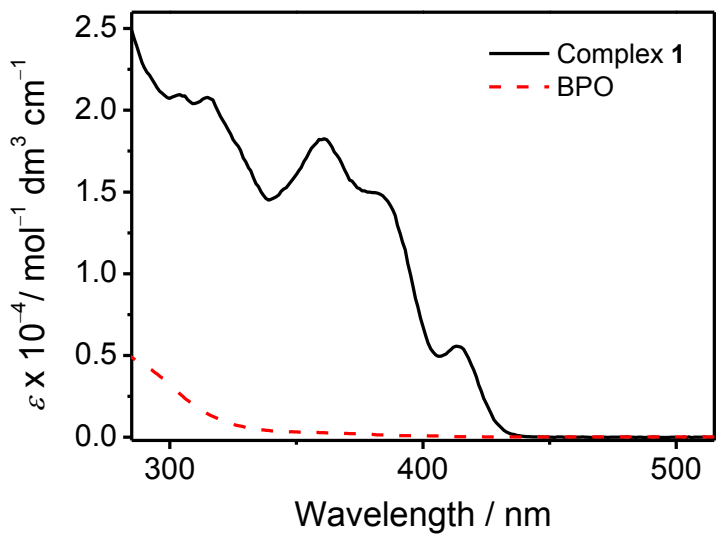

Figure S3. UV-Vis absorption spectra of complex $\mathbf{1}$ and 2-(triisopropylsilylethynyl)benzo $[b]$ phosphole $P$-oxide $\quad$ (BPO) in dichloromethane solution. 


\section{Memory Performances}

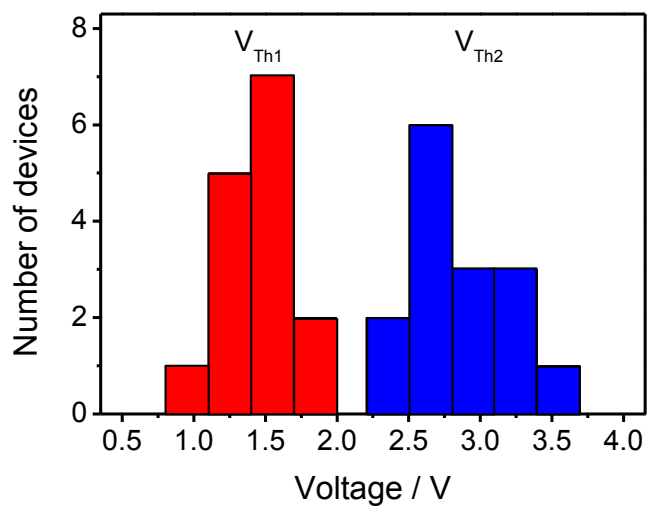

Figure S4. The distribution of two switching threshold voltages ( $V_{\mathrm{Th} 1}$ and $\left.V_{\mathrm{Th} 2}\right)$ among 15 devices.

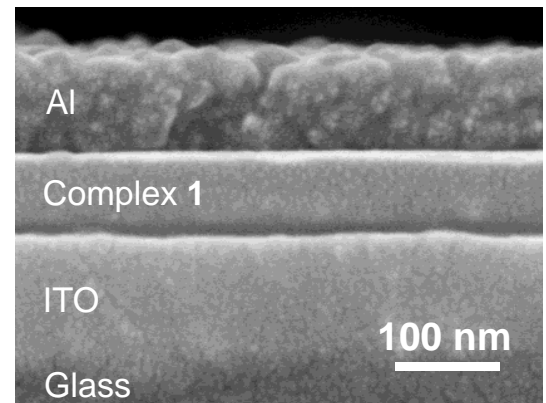

Figure S5. SEM image of a cross-section of the ITO/complex $1(80 \mathrm{~nm}) / \mathrm{Al}$ device.

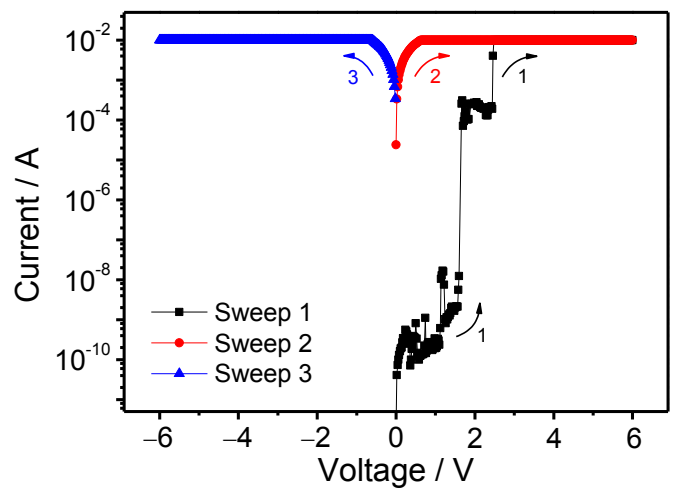

Figure S6. Current-voltage characteristics of the ITO/complex $\mathbf{1}(80 \mathrm{~nm}) / \mathrm{Al}$ device under a positive voltage bias. 


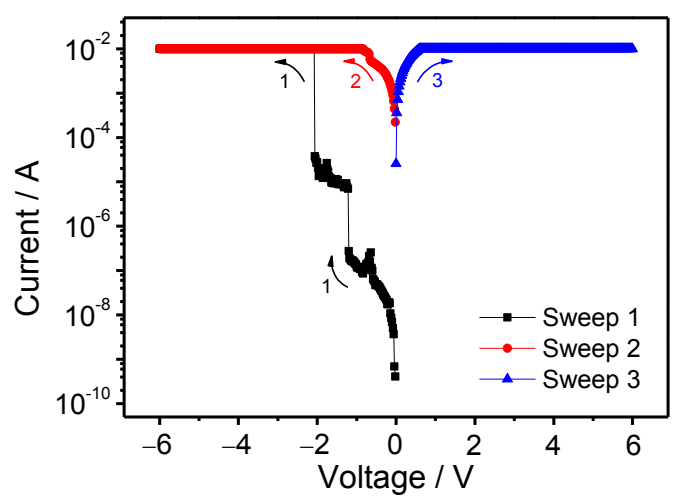

Figure S7. Current-voltage characteristics of the ITO/complex $\mathbf{1}(80 \mathrm{~nm}) / \mathrm{Al}$ device under a negative voltage bias. 


\section{NMR Spectra}

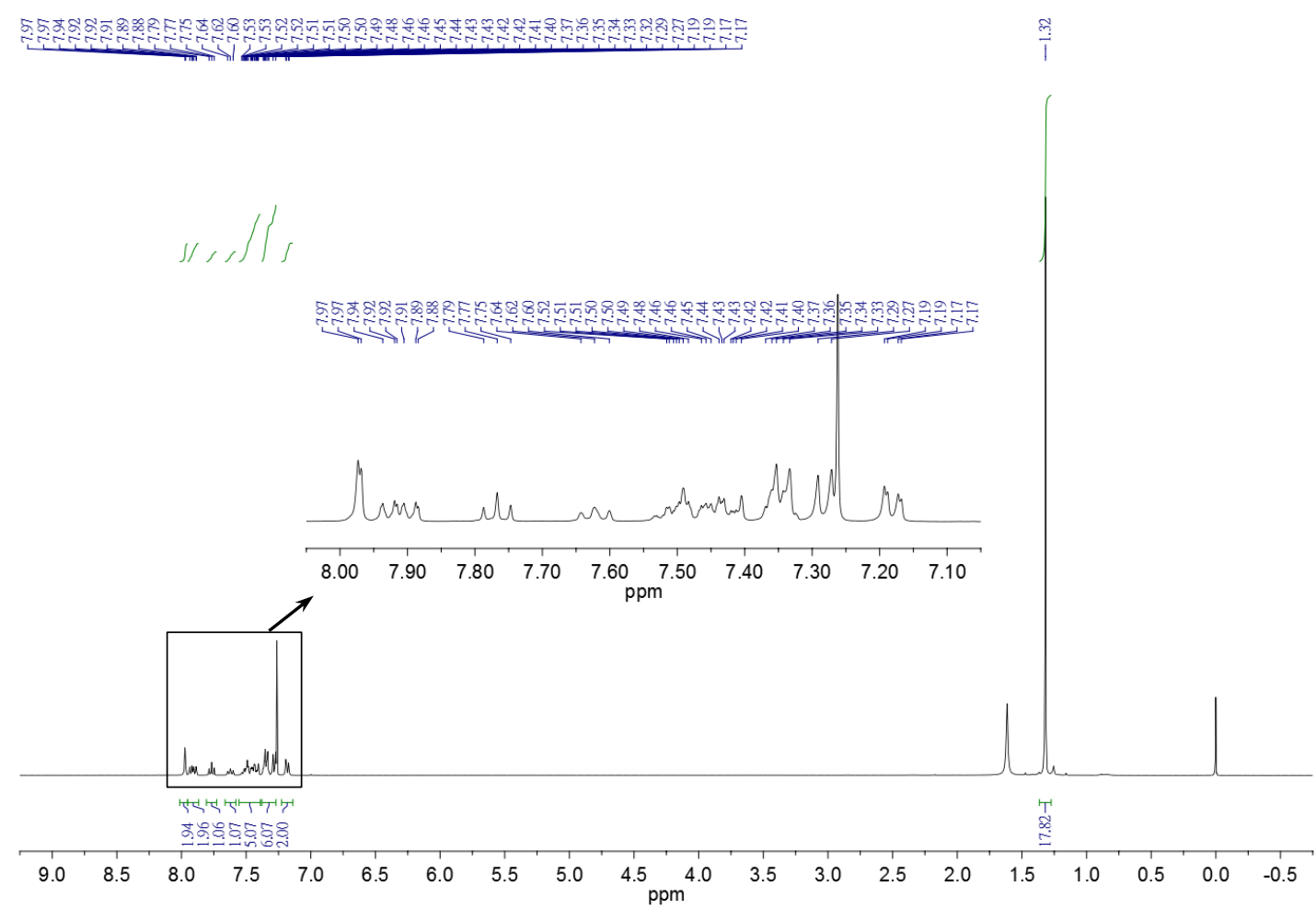

Figure S8. $\quad{ }^{1} \mathrm{H}$ NMR spectrum of complex 1 in $\mathrm{CDCl}_{3}$.

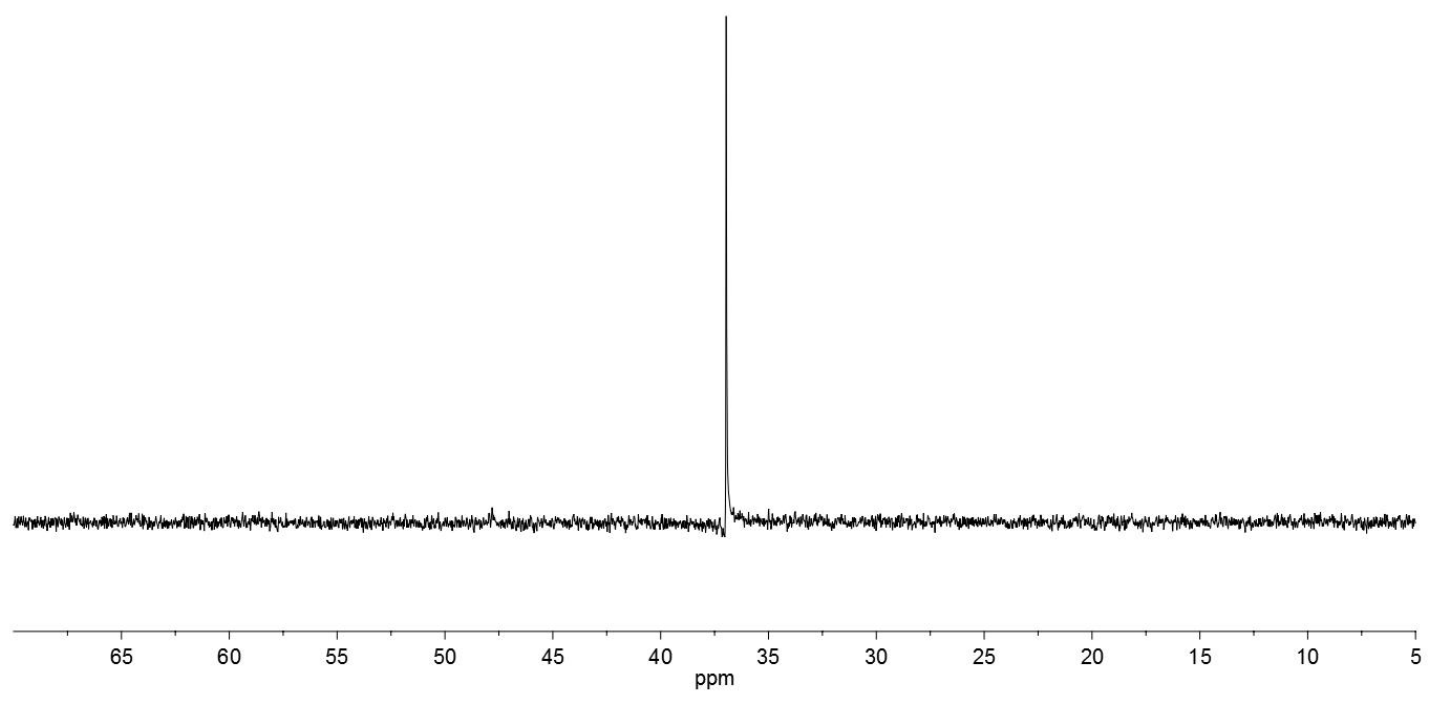

Figure S9. $\quad{ }^{31} \mathrm{P}\left\{{ }^{1} \mathrm{H}\right\}$ NMR spectrum of complex $\mathbf{1}$ in $\mathrm{CDCl}_{3}$. 


\section{References}

1 Wong, K. M.-C.; Hung, L.-L.; Lam, W. H.; Zhu, N.; Yam, V. W.-W. J. Am. Chem. Soc. 2007, 129, 4350.

2 Matano, Y.; Hayashi, Y.; Suda, K.; Kimura, Y.; Imahori, H. Org. Lett. 2013, 15, 4458.

3 Demas, J. N.; Crosby, G. A. J. Phys. Chem. 1971, 75, 991.

4 Van Houten, J.; Watts, R. J. Am. Chem. Soc. 1976, 98, 4853.

5 Connelly, N. G.; Geiger, W. E. Chem. Rev. 1996, 96, 877.

6 Yam, V. W.-W.; Wong, K. M.-C.; Hung, L.-L.; Zhu, N. Angew. Chem. Int. Ed. 2005, 44, 3107. 